\title{
Biventricular Pacing in Congestive Heart Failure: Follow-up Data
}

\author{
N Naik, R Yadav, R Juneja, A Roy, S Anandraja, KK Talwar.
}

Biventricular (BV) pacing is a promising option for patients with advanced heart failure and electromechanical conduction delay. We present our experience in 17 patients. 19 patients underwent the implant procedure at our institute: 3 others had their implant elsewhere and were on our follow-up. The mean age was $46.6+10.7$ years and 14 were males. A conventional indication for pacing was present in $8 ; 4$ of them had previously received a pacemaker. All had severe LV dysfunction. The implant procedure was successful in 12/14- in one the CS lead could not be anchored satisfactorily and a dual chamber device was inserted. Another patient required an epicardial lead due to CS dissection. On a follow-up of 2-38 months, significant improvement in NYHA class, LV dimensions and function, and 6-minute walk test were seen. Three patients worsened immediately after BV pacing - all had their leads implanted in the great cardiac vein (GCV). An epicardial LV lead was implanted in one of these patients. Five patients died during follow-up, including all 3 who had CS leads in the GCV. The cause of death was worsening $\mathrm{CHF}$ in 3 and $\mathrm{SCD}$ in 2. One patient had worsening CHF due to CS lead dislodgement following DC version for VT. The patient subsequently underwent lead repositioning. In conclusion, BV pacing is associated with clinical and hemodynamic improvements along with reversal of LV remodeling. Pacing from GCV maybe associated with clinical deterioration. 